\title{
An improved perturbation method of photovoltaic power generation MTTP

\author{
Yunjian Li
}

Department of North China Electric Power University, Beijing, 102206, China

\begin{abstract}
Keywords: photovoltaic power generation, maximum power point tracking, improved perturbation
\end{abstract} method.

\begin{abstract}
At In the photovoltaic, maximum power point tracking technology is an inevitable problem. The perturbation method is simple to operate, easy to implement and so on, however, the traditional perturbation method tracks slowly and is easy to shake. This paper compares the tracking characteristics between long step and short step of traditional perturbation method and combines the two to put forward an improved perturbation method for fast, smooth tracking.
\end{abstract}

\section{Introduction}

In recent years, the energy shortage has become a major worldwide problem. The overexploitation and uncontrolled use of fossil energy, including coal, oil and natural gas, not only make energy crisis more intense, but also cause great damage on the human environment. Using clean, reliable and renewable energy has become a coincident goal of the world. Solar photovoltaic power generation has become a focus for its stable reliable long life, convenient installation and maintenance. In the photovoltaic power generation, energy changes from luminous energy into electric energy by the Photovoltaic Array, but the relationship between power and voltage is nonlinear, which means that there is a maximum power point in every moment [1-3]. So, how to further improve the efficiency of solar cells and make full use of photovoltaic arrays to convert energy has become an important research direction of the solar photovoltaic power generation system. In order to take full advantage of solar energy and get the maximum power output, we must track and control the maximum power point of the Photovoltaic Array [4, 5].

\section{The model and output characteristics of photovoltaic cells}

\section{Model}

In general, a photovoltaic cell equivalent circuit is like Figure 1. According to the photovoltaic effect, PN junction can be equivalent to the reverse parallel of an ideal current source and a diode. $R_{0}$ represents the resistance load. The various losses in the circuit can be represented by a resistor $R_{2}$ in series with the load resistor $R_{0}$. Meanwhile, due to the leakage current of the solar cell, the current flowing into the load will be decreased, we need to use a parallel resistor $R_{1}$ to represent.

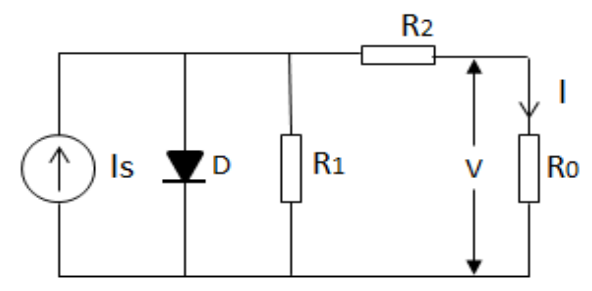

Fig.1: Model

In Figure 1, $I_{s}$ indicates the photocurrent. The stronger the light intensity is, the greater the current $I_{s}$ will be. Part of the photocurrent flows into the diode, the other part flows into the load. According to the diode operating characteristics we know:

$$
I=I_{s}-I_{0}\left\{\exp \left[\frac{q\left(V+R_{2} I\right)}{A K T}\right]-1\right\}-\frac{V+R_{2} I}{R_{1}}
$$


Therefore, the output power of the photovoltaic cell is:

$$
P=I V
$$

In the above formula, A represents the PN junction coefficient, $\mathrm{K}$ represents Boltzmann's constant, and q represents the charge constant. In order to simplify, we use the equivalent model of photovoltaic cells under ideal conditions. In Figure $1, R_{1}$ is infinite, $R_{2}$ is equal to zero.

Therefore, the above formula can be simplified to:

$$
P=I_{s} V-I_{0} V\left\{\exp \left[\frac{q V}{A K T}\right]-1\right\}
$$

From the above equation we can observe that the output power of photovoltaic cells and the voltage across the load is non-linear.

\section{Characteristics.}

Seen from the equation, P relate to temperature, light and voltage across the load. When the temperature and light are constant, the operating characteristics of photovoltaic cell is shown in Figure 2.

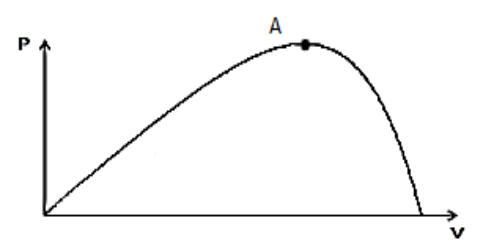

Fig.2: Characteristic

A is the maximum power point. Our goal is to use fast, stable, and reliable algorithm to achieve maximum power point tracking in a changing environment. Figure 3 is a $P-V$ curve at the same temperature for different lighting conditions. Figure 4 is a photovoltaic characteristics under different temperature conditions in the same light.

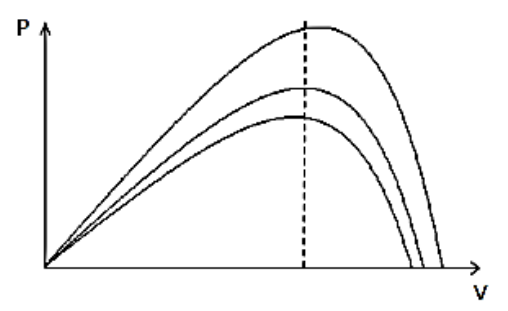

Fig.3: Temperature $=\mathrm{C}$

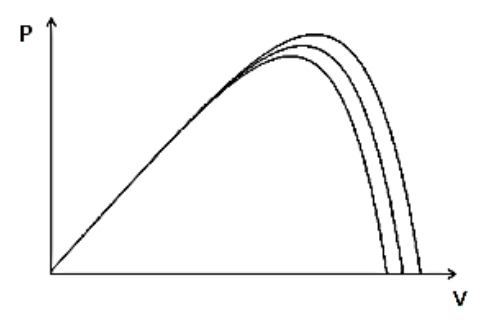

Fig.4: Light $=\mathrm{C}$

From the above, we can see that there is only one pole of the photovoltaic characteristic curve, which is called the maximum output power point. In the left side of the maximum power point, the output power increases monotonically with the increasing voltage across the load, $\Delta p / \Delta v>0$. In the right side of the maximum power point, the output power decreases monotonically with the decreasing voltage across the load, $\Delta \mathrm{p} / \Delta \mathrm{v}<0$. In the maximum power point, $\Delta \mathrm{p} / \Delta \mathrm{v}=0$. 


\section{Traditional perturbation method of the maximum power point tracking and the weaknesses analysis}

\section{Feature}

Below is a flow chart of the traditional perturbation method be transformed into a multi-objective single-level optimization problem :

Using MATLAB software, we can get the maximum power point tracking simulation under perturbation method when $\Delta \mathrm{d}=0.4$ and $\Delta \mathrm{d}=4$.

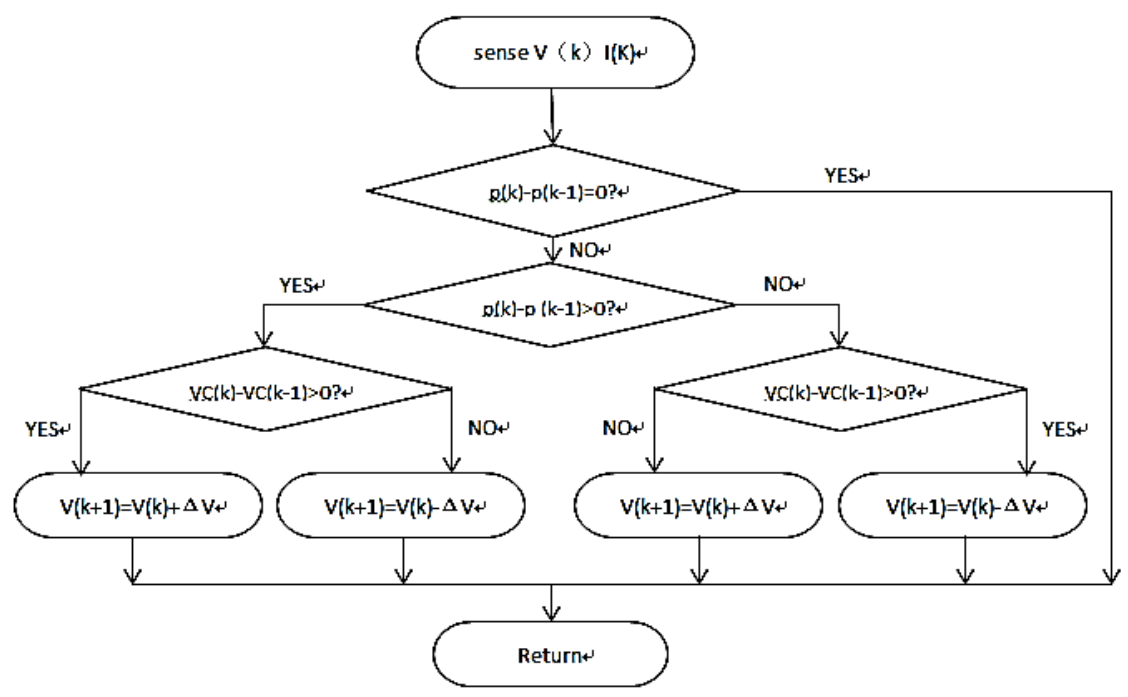

Fig.5: Flowchart

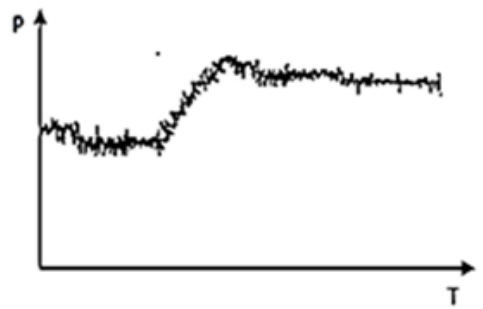

Fig.6: $\Delta d=0.4$

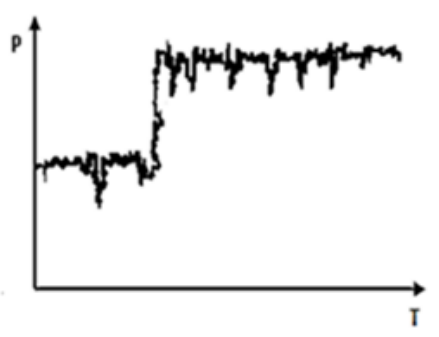

Fig.7: $\Delta d=4$

Through the figure we can get the following conclusions:

i) It can be roughly divided into two types of PV curve working condition. Slowly varying smooth working condition and sudden changes, volatility jump status.

ii) For small step perturbation method, it can be better adapted to the first operating state, but for the sudden jump in working condition, it responds slowly.

iii) For a long step perturbation method, it can be better adapted to the second operation, rapid response, but for gentle working condition, it will produce a lot of unwanted jitter and reduce the efficiency of photovoltaic power generation. 


\section{Weakness}

Traditional method have phenomenon out of control.

As shown in Fig.8. According to the normal working principle, the working condition on curve 1 will move from point A to A'. However, if the sun light increases suddenly. it will move from A on curve 1 to $\mathrm{B}$ on curve 2 .According to perturbation method, we judge that $\Delta \mathrm{p}>0$, so $\mathrm{V}>0$, and the working point is in the left side of the maximum power point, the voltage should be further reduced. At this time, if the light intensity increased further, the work point will move to the curve 3. Similarly, we will still get the wrong conclusion that power should be further increased. The maximum power point tracking will be out of control.

We can solve this disadvantage by increasing the frequency of our perturbation and reducing disturbance step.

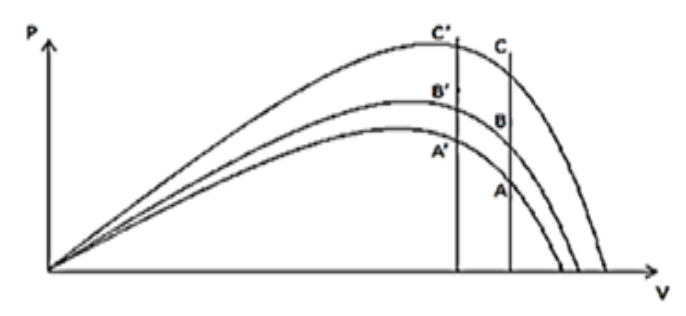

Fig.8 Weakness

\section{Improved perturbation method and its simulation result}

We know that long step in the jump working condition has a good tracking effect, at the same time, short step in the smooth working condition has a good tracking effect. As a result, the improved method is to combine big step and small step. Fig.9 is the flow chart of the improved perturbation method.

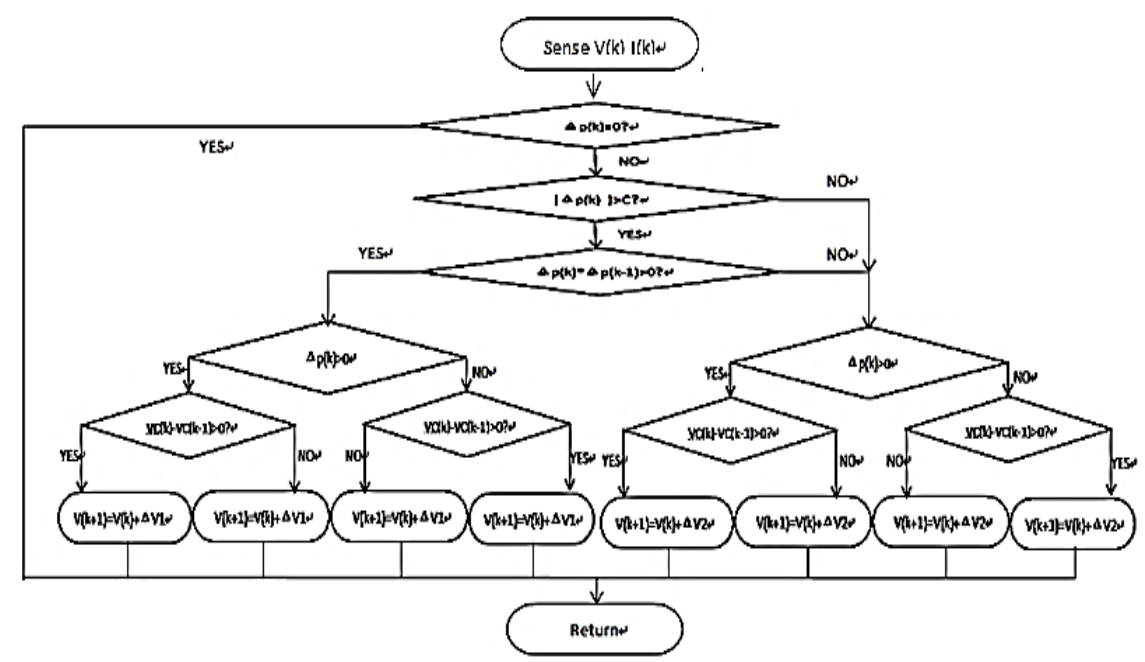

Fig.9 New flow chart

The idea of improved perturbation method is:

We define a new discriminant value C. On this basis, it can be divided into long step disturbance tracking and short step disturbance adjusting to adapt to various working state. First of all, it is easy to fast track by using long step disturbance. To determine whether it is just across the maximum power point, we should compare $\Delta P_{(\mathrm{i})}$ an $\Delta P_{(\mathrm{i}-1)}$. If they are of the same number, it is still working on the same side after disturbance .Otherwise, it just across the maximum power point. Then we need to change long step into short step, and disturbance to the opposite direction.Every time we need to compare the absolute of the difference and $\mathrm{C}$ to determine 
whether there is a the sudden jump working condition, when $\Delta \mathrm{p}<\mathrm{C}$, the system is working in the smooth condition.

On the contrary, it means that during the two disturbances the working state changes dramatically, the working condition changed from $A$ to $B$. We need to transfer small step disturbance to the long step disturbance, same with the original perturbation step, we go back to the fast mutation track working state. In summary, we can take care of both working condition through in is way .

Using MATLAB software, we can get Fig.10, which is the maximum power point tracking simulation under improved perturbation.

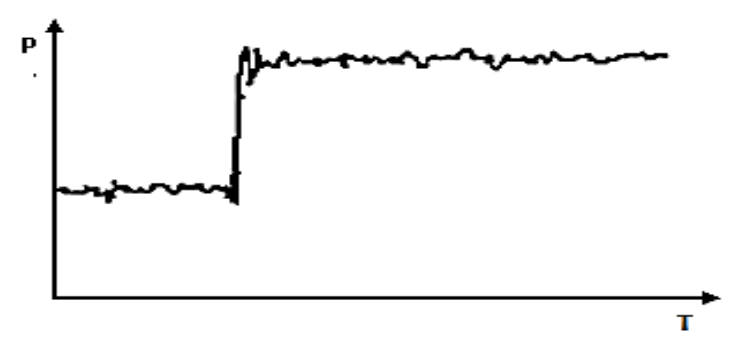

Fig.10 The simulation result of the improved perturbation method

\section{Conclusions}

The improved perturbation method combines the advantages of both long and short step. At the same time, it is better to avoid the runaway of original perturbation method. Which is advanced and practical.

\section{References}

[1] C.Zhang: The study of Grid-connected photovoltaic system MTTP and islanding detection technology (Ph.D., Zhejiang University, China 2006), p.24.

[2] Zhigang Fang, Xuexun Guo, Lin Xu and Han Zhang. Experimental Study of Damping and Energy Regeneration Characteristics of a Hydraulic Electromagnetic Shock Absorber. Advances in Mechanical Engineering, vol. 2013, (2013. 05) Article ID 943528.

[3]Information on: http://baike.baidu.com/link?url=foi0lns9SrmtacqOgqC2EI6 R36wd_7mOys YsgMg2 SnvaX9s Mg0b0mSE1lVBICZb_cT10JrICeA_uwefeWmmvo_

[4] Information on http://wenku.baidu.com/view/9614e31114791711cc791708. html

[5] Shuang Liang, Xuehao Hu, Dongxia Zhang, et al. Probabilistic models based Evaluation method for Capacity Credit of Photovoltaic Generation. Automation of Electric Power Systems, vol. 36 (2012) no. 13, p. 32-37. 